\title{
Importance of Precise Radial Velocities for Cepheid Binaries
}

\section{Szabados}

Konkoly Observatory of the Hungarian Academy of Sciences, P.O. Box 67, H-1525 Budapest, XII, Hungary

\begin{abstract}
In view of the high incidence of duplicity among Cepheids, a large number of binary systems remain to be discovered because of the low amplitude of the orbital effect superimposed on the radial velocity variation due to radial pulsation. This fact is shown by simple statistics. The importance of combining astrometric data with radial velocity measurements is also emphasized. Finally the systematics of the radial velocity amplitude as a function of the pulsation period is considered for classical Cepheids.
\end{abstract}

\section{Introduction}

Radial velocity observations of classical Cepheids are of increasing importance in the light of the recent results on these radially pulsating variables.

Progress in astronomicai spectroscopy - especially the improvement in the precision of radial velocity data (see e.g. the extensive series obtained by Bersier et al. (1994); Pont, Burki, \& Mayor (1994); Gorynya et al. (1996) and references therein; Imbert (1996)) and the development of ultraviolet spectroscopy, especially utilizing the IUE satellite (see e.g. Evans 1992a) - has led to the discovery that a considerable fraction of Cepheids belong to binary and multiple systems.

Because Cepheids are primary distance indicators, calibration of the zeropoint of their period-luminosity (or $\mathrm{P}$ - $\mathrm{L}$-color) relation is essential, and the use of Cepheids members in binary systems gives an impetus to these studies, since the luminosity of these particular Cepheids can be determined from the properties of the binary system (see e.g. Evans 1992b).

\section{On the ample occurrence of binaries among Cepheids}

An extensive study of the photometric and radial velocity data of classical Cepheids, based on all the information available in the literature, has resulted in finding a high incidence of binaries among Cepheids, and even a selection effect in revealing their binary nature was pointed out (Szabados 1995). The selection effect manifests itself as a lower frequency of known binaries towards fainter Cepheids, as a consequence of insufficient observation of these stars.

Quite recently, however, the fainter Cepheids also became suitable targets for radial velocity studies which contributed to the reduction of the selection effect but its complete removal has not been achieved yet. The comparison 

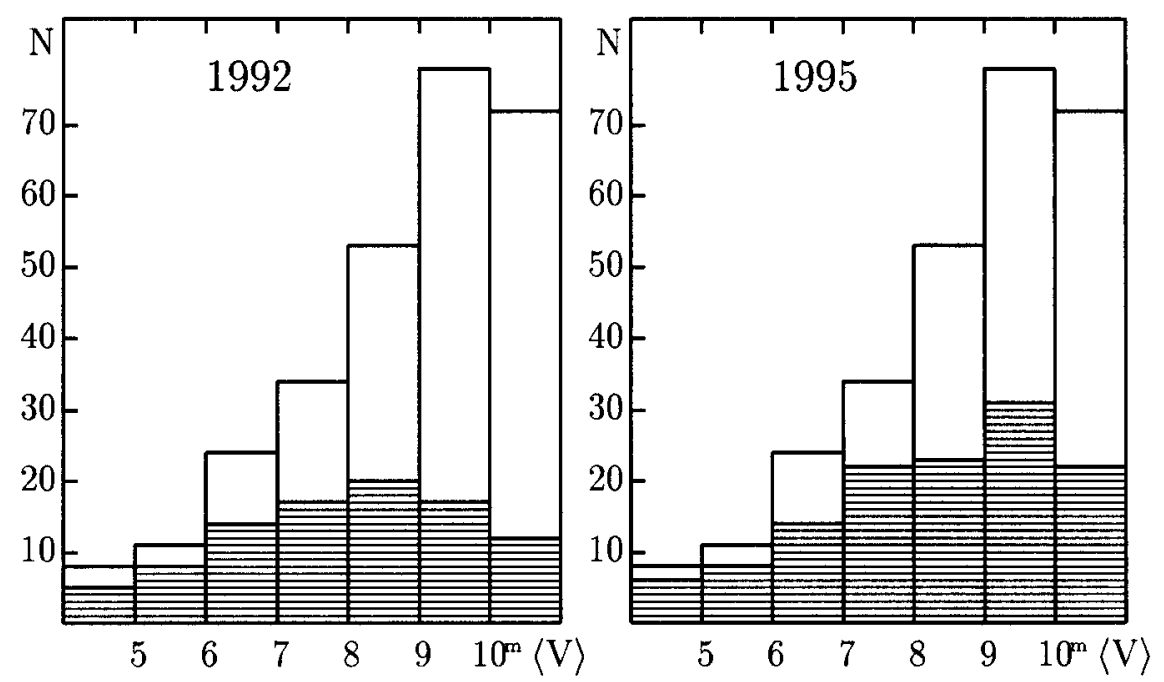

Figure 1. The brightness dependence of "occurrence" of binaries among Cepheids. The hatched area represents the binaries, while the empty rectangle is the rest of the sample, including the unrecognized binaries. The abscissa is the mean brightness in Johnson V-band. A definite progress is seen in the discovery of new SB-Cepheids when comparing the situation in 1992 and 1995 but a number of spectroscopic binaries remain to be revealed among the Cepheids fainter than the eighth magnitude.

of the situation in 1992 and 1995 is shown in Figure 1. In the last few years we are witnessing further progress (e.g. more than two dozen new spectroscopic binaries have been discovered among Cepheids by Szabados (1996) and Szabados $\&$ Pont (1998) in the 7.2-12.4 visual-magnitude range but the final goal is to include the faint (10-13 magnitude in $\mathrm{V}$-band, or possibly even fainter) Cepheids in thorough radial velocity surveys.

Precise measurements are essential for performing a reliable decomposition of the radial velocity variation into pulsational and orbital components. The top left panel of Figure 2 shows that Cepheids with known orbital elements typically have $1-5$ year orbital periods, i.e. observational projects of considerable duration are necessary. It is evident from the top right panel of Figure 2 that the orbital elements have been successfully determined for the systems whose orbital effect (the peak-to-peak amplitude of the orbital radial velocity variation, $2 K$ ) generally exceeds $10 \mathrm{~km} \mathrm{~s}^{-1}$. A similar histogram is constructed for those Cepheids whose membership in binary systems has been established but no orbital solution is available yet. As is seen in the bottom panel of Figure 2, the tendency is just the opposite to what is seen for the "solved" systems. Here $\Delta v_{\gamma}$ is the range of the observed $\gamma$-velocity variation being only a lower limit for the orbital effect. The conclusion to be drawn based on this latter histogram is obvious - most of the spectroscopic binaries among Cepheids have small- 


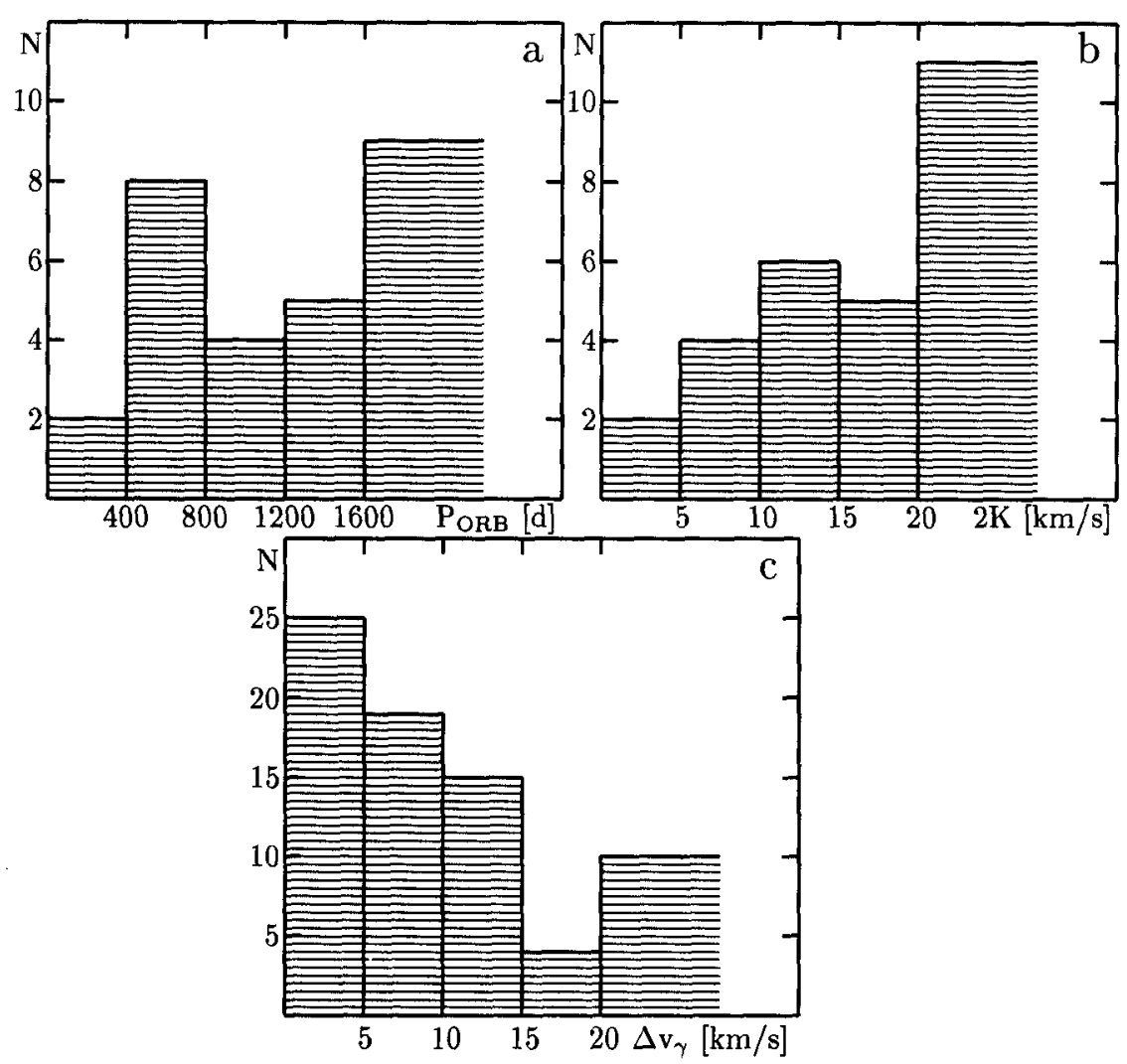

Figure 2. a: Distribution of the orbital periods; b: distribution of the orbital effect; c: distribution of the partial orbital effect for the SB-Cepheids with unsolved orbits

amplitude orbital effects. Therefore their orbital elements can be calculated if precise radial velocities are available throughout a complete orbital cycle.

High-precision measurements are not the only requirement; the number of data and their temporal distribution is also crucial for the successful separation into pulsational and orbital effects. In addition, other subtle effects can be superimposed on the complex radial velocity variation: e.g. Dinshaw et al. (1989) suspected the presence of starspots on the surface of Polaris, and quite recently Butler (1998) was able to point out low-amplitude variability in the radial velocities of non-pulsating stars in the Cepheid instability strip. Because of the large-scale pulsational motion, it is a great observational challenge to reveal such variability (whose nature has not been clarified yet, but enhanced chromospheric activity is a promising alternative) or to refute the presence of such an effect on the stars which do pulsate. Vinkó, Kaszás \& Kiss (1999) argue that the phase-dependent line asymmetry is an obstacle that makes the discovery of non-pulsational effects very difficult from the radial velocity data because the 
velocity determined from the spectral lines depends on the lines used and the technique for measuring lines.

\section{Hipparcos parallaxes and Cepheids in binary systems}

As it was pointed out (Szabados 1997a), Hipparcos parallaxes of Cepheids belonging to binary systems may have been falsified by neglecting the angular displacement of the Cepheid due to the orbital motion around the mass center of the system. In fact, the period-luminosity relation can be based only on the measured parallax of the solitary Cepheids and the true (i.e. properly corrected) parallax of binary Cepheids.

Whether the orbital motion can be observed by astrometric and/or spectroscopic means depends on the inclination of the orbital plane. A slight $\gamma$-velocity variation may indicate that the orbital plane of the binary is almost perpendicular to the line of sight. In the case of the supergiant Cepheids even the shortest orbital periods mean that the angular displacement due to orbital motion can be commensurable with the trigonometric parallax itself (Szabados 1997a).

Therefore precise radial velocities help select Cepheids whose parallax may be distorted by binarity and these objects are essential targets for both groundbased interferometric projects and the next-generation microarcsec satellites. U Sagittarii is an immediate candidate (Szabados 1997b).

\section{The period-amplitude relation from radial velocity data}

The systematic behavior of Cepheids as a function of the pulsation period is well known. The dependence of the amplitude of the brightness variation on the pulsation period is amply discussed in the literature (see e.g. Eichendorf \& Reinhardt (1977) and references therein) but the radial-velocity amplitude vs. pulsation period relation is very much neglected. The most detailed analysis (Kovács, Kisvarsányi, \& Buchler 1990) was based on only 57 Cepheids.

Now a thorough study has been performed involving about 300 Galactic Cepheids. Some of the results are briefly mentioned here.

The systematic progression of the radial velocity amplitude is seen in Figure 3. The deviating points in the large-amplitude region are the Cepheids which belong to binary systems and (because of insufficient data) whose orbital $\gamma$-velocity variation has not yet been separated from the amplitude of pulsational changes. Based on this feature, AY Sgr (at $\log P=0.818$ ) and SY Nor (at $\log P=1.102$ ) belong to spectroscopic binary systems, and therefore their radial velocity variations deserve a thorough study.

Otherwise the pattern in this diagram is similar to that shown by the luminosity amplitude. The drop in amplitude at a pulsation period of about ten days - a characteristic feature in the amplitude of brightness variation vs $\log P$ diagrams - is very pronounced here. The group of the low-amplitude s-Cepheids stands out clearly, too.

As to the normal amplitude Cepheids, i.e. the fundamental-mode pulsators, the long-period Cepheids are characterized by larger radial velocity amplitudes than their counterparts with periods shorter than 10 days. This behavior also re- 


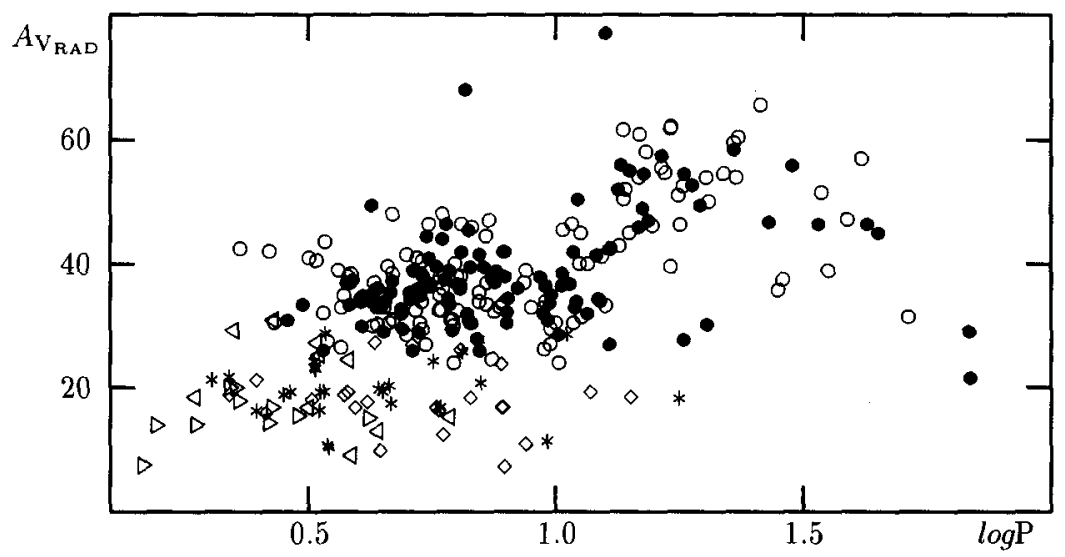

Figure 3. The amplitude of the pulsational radial velocity variation (in $\mathrm{km} \mathrm{s}^{-1}$ ) as a function of the pulsation period. The various symbols denote: ० - normal amplitude solitary Cepheids; $\bullet$ - normal amplitude Cepheids with companion; $\diamond$ - solitary s-Cepheids; $*-$ - -Cepheids with known companion; $\triangleleft$-fundamental mode oscillation of beat Cepheids; $\triangleright$ - first overtone of beat Cepheids

sembles the pattern typical of the systematics in light-variation. The amplitude ratio of the radial velocity and luminosity variations is, however, different for the short-period and long-period pulsators. This behavior and its implications will be discussed in a forthcoming paper.

\section{Conclusion}

Precise radial velocity measurements of Cepheids and especially of binary systems among them can help to determine the physical properties of the individual stars, which is a necessary step in attempts to perform an exact calibration of the zero-point of the P-L relation. The number of calibrating Cepheids can be increased via the thorough study of Cepheids with known spectroscopic companions. In addition, systematic radial-velocity studies of faint Cepheids will result in discovering numerous SB-systems unrecognized so far.

Acknowledgments. The author has profited very much from the discussions with Drs. Mária Kun and József Vinkó. This work was supported in part by Hungarian OTKA-projects T 014852 and T 022946 as well as AKP 97-58 project. The author acknowledges the IAU travel grant and OTKA U 28572 grant that made possible his participation in the conference. 


\section{References}

Bersier, D., Burki, G., Mayor, M., \& Duquennoy, A. 1994, A\&AS, 108, 25

Butler, R.P. 1998, ApJ, 494, 342

Dinshaw, N., Matthews, J.M., Walker, G.A.H., \& Hill, G.M. 1989, AJ, 98, 2249

Eichendorf, W., \& Reinhardt, M. 1977, A\&A, 61, 827

Evans, N.R. 1992a, ApJ, 384, 220

Evans, N.R. 1992b, ApJ, 389, 657

Gorynya, N.A., Samus, N.N., Rastorgouev, A.S., \& Sachkov, M.E. 1996, Pis. AZh, 22, 198

Imbert, M. 1996, A\&AS, 116, 497

Kovács, G., Kisvarsányi, E.G., \& Buchler, J.R. 1990, ApJ, 351, 606

Pont, F., Burki, G., \& Mayor, M. 1994, A\&AS, 105, 165

Szabados, L. 1995, in Astrophysical Applications of Stellar Pulsation (ASP Conf. Ser., 83), R.S. Stobie \& P.A. Whitelock, San Francisco: Astron. Soc. Pac., 357

Szabados, L. 1996, A\&A, 311, 189

Szabados, L. 1997a, in Hipparcos Venice '97 (ESA SP-402) , B. Battrick, Noordwijk: European Space Agency, 657

Szabados, L. 1997b, in Pulsating Stars - Recent Developments in Theory and Observation, D.D. Sasselov and M. Takeuti, Tokyo: Universal Academy Press, 81

Szabados, L., Pont, F. 1998, A\&AS, 133, 51

Vinkó, J., Kaszás, G., \& Kiss, L.L. 1999, these Proceedings

\section{Discussion}

Griffin: We have heard several times this morning of the need for better radial velocities, accurate amplitudes of secondaries, accurate dynamical masses, to precisions that we spectroscopists simply cannot yet achieve. Spectral lines in many of these cases are asymmetrical, varying, or badly confused with other spectra, and so on. This is not an easy matter; we do what we can, but we have to contend with what nature provides. Yes, we would like to provide you with accurate velocities for Cepheids, indeed for all these difficult cases; we would like to obtain them for ourselves. Perhaps more support voiced by the rest of the community would help us to make more substantial progress faster.

Lampens: In the comparison of ground-based versus Hipparcos parallaxes, it would be good to use the error bars (of the same level as the plotted parallaxes) to compare between single and binary Cepheids because, in general, the errors are larger in the case of double and multiple systems. In some cases, also, re-reduction with input of new information would be extremely welcome.

Szabados: In the case of binary Cepheids the larger error is simply caused by the fact that the apparent orbital motion. i.e. the displacement on the celestial 
sphere, has not been taken into account, as it has been considered as an extra error source in the data reduction. 